AIP Applied Physics

\title{
Influence of alloy inhomogeneities on the determination by Raman scattering of composition and strain in Si1-xGex/Si(001) layers
}

\author{
J. S. Reparaz, I. C. Marcus, A. R. Goñi, M. Garriga, and M. I. Alonso
}

Citation: J. Appl. Phys. 112, 023512 (2012); doi: 10.1063/1.4737486

View online: http://dx.doi.org/10.1063/1.4737486

View Table of Contents: http://jap.aip.org/resource/1/JAPIAU/v112/i2

Published by the American Institute of Physics.

\section{Related Articles}

Monitoring the in-situ oxide growth on uranium by ultraviolet-visible reflectance spectroscopy J. Appl. Phys. 112, 093104 (2012)

Experimental investigation of photonic band gap influence on enhancement of Raman-scattering in metaldielectric colloidal crystals

J. Appl. Phys. 112, 084303 (2012)

Design of lumpy metallic nanoparticles for broadband and wide-angle light scattering

Appl. Phys. Lett. 101, 141112 (2012)

Experimental surface-enhanced Raman scattering response of two-dimensional finite arrays of gold nanopatches Appl. Phys. Lett. 101, 111606 (2012)

Origins of high visible light transparency and solar heat-shielding performance in LaB6

Appl. Phys. Lett. 101, 041913 (2012)

\section{Additional information on J. Appl. Phys.}

Journal Homepage: http://jap.aip.org/

Journal Information: http://jap.aip.org/about/about_the_journal

Top downloads: http://jap.aip.org/features/most_downloaded

Information for Authors: http://jap.aip.org/authors

\section{ADVERTISEMENT}

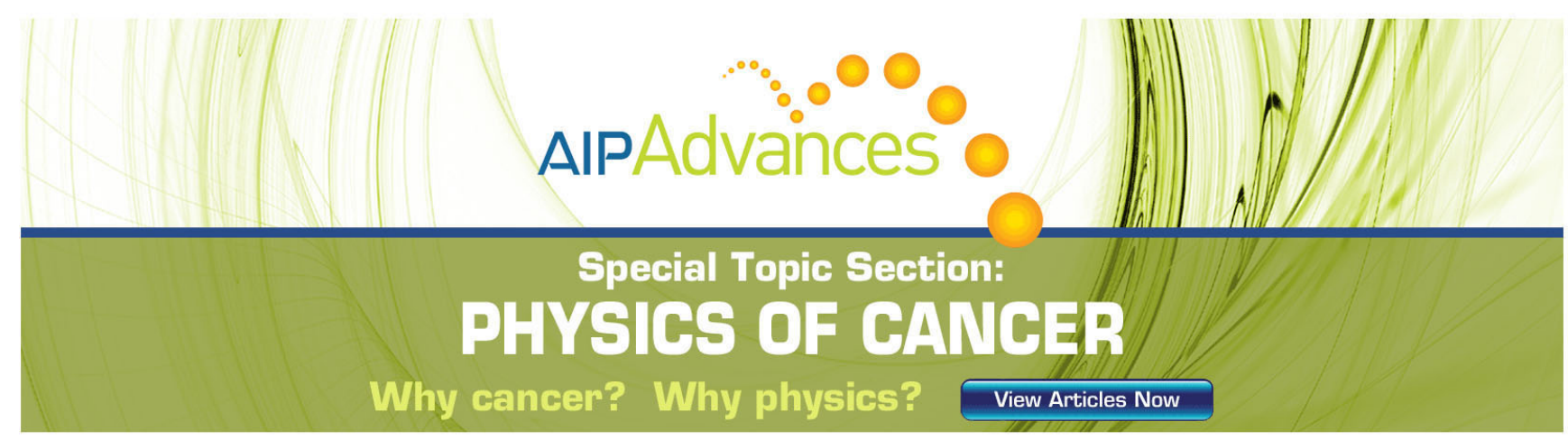




\title{
Influence of alloy inhomogeneities on the determination by Raman scattering of composition and strain in $\mathrm{Si}_{1-x} \mathrm{Ge}_{x} / \mathrm{Si}(001)$ layers
}

\author{
J. S. Reparaz, ${ }^{1}$ I. C. Marcus, ${ }^{1}$ A. R. Goñi,,${ }^{1,2}$ M. Garriga, ${ }^{1}$ and M. I. Alonso ${ }^{1}$ \\ ${ }^{1}$ Institut de Ciència de Materials de Barcelona (ICMAB-CSIC), Esfera UAB, 08193 Bellaterra, Spain \\ ${ }^{2}$ ICREA, Passeig Lluís Companys 23, 08010 Barcelona, Spain
}

(Received 20 December 2011; accepted 20 June 2012; published online 20 July 2012)

\begin{abstract}
In this work, we investigate the influence of alloy composition inhomogeneities on the vibrational properties of strained $\mathrm{Si}_{1-x} \mathrm{Ge}_{x} / \mathrm{Si}$ layers with $x$ ranging from 0 to 0.5 . We show that the frequencies of the principal alloy vibrational modes ( $\mathrm{Ge}-\mathrm{Ge}, \mathrm{Si}-\mathrm{Ge}$, and $\mathrm{Si}-\mathrm{Si}$ ) are strongly influenced by the distribution of Ge atoms within the alloy layers, which becomes gradually random following a series of sequential annealing steps. Our measurements suggest that the composition dependence of the optical phonon frequencies in fully random and unstrained alloys is well described by the results previously published by Alonso and Winer [Phys. Rev. B 39, 10056 (1989)]. In the general case of an alloy layer with unknown degree of compositional inhomogeneity and/or strain relaxation, though the analysis of the Raman spectra is not straightforward. Therefore, we propose an analytical/graphical method to accurately estimate the Ge content and residual strain of SiGe layers exhibiting any level of compositional disorder or strain status, by performing a single Raman measurement. This would be extremely useful in situations where x-ray measurements cannot be conducted. We show that our procedure to treat the Raman data holds for the whole compositional range but with different accuracy depending upon the case: (i) For annealed SiGe layers (mostly strain relaxed) the Ge content $x$ can be directly determined with high accuracy of \pm 0.01 . (ii) For strained samples (usually as-grown samples) an extra criterion must be adopted seeking for a graphical solution, accounting for the degree of compositional inhomogeneity. In this case, the error in the determination of Ge content depends on alloy composition, being the upper bound \pm 0.02 for $x<0.3$ and \pm 0.03 for $x>0.3$. (C) 2012 American Institute of Physics. [http://dx.doi.org/10.1063/1.4737486]
\end{abstract}

\section{INTRODUCTION}

In the last two decades, strained $\mathrm{SiGe/Si}$ alloy layer structures attracted increasing attention due to their higher performance as active electronic components. Larger carrier mobilities, ${ }^{1,2}$ higher device-switching speeds, ${ }^{3-5}$ and lower power consumption ${ }^{6}$ are some of the advantages that make strained $\mathrm{SiGe} / \mathrm{Si}$ structures a good alternative to their already well established counterpart. Successful inclusion of Ge in a great variety of device architectures such as heterojunction bipolar transistors ${ }^{7-9}$ (HBTs), metal-oxide-semiconductor field-effect transistors ${ }^{10-12}$ (MOSFETs), and optical modulators $^{13,14}$ has been already clearly demonstrated. The advantages offered by this material system partly originate from the larger carrier mobilities ${ }^{1}$ exhibited by Ge $\left(\mu_{e}=3900 \mathrm{~cm}^{2} / \mathrm{Vs}\right.$, $\left.\mu_{h}=1800 \mathrm{~cm}^{2} / \mathrm{Vs}\right)$ as compared to $\mathrm{Si}\left(\mu_{e}=500 \mathrm{~cm}^{2} / \mathrm{Vs}\right.$, $\left.\mu_{h}=1450 \mathrm{~cm}^{2} / \mathrm{Vs}\right)$. Furthermore, the larger lattice constant of Ge $(a=5.658 \AA)$ relative to that of $\mathrm{Si}(a=5.431 \AA)$ usually leads to a built-in strain which further affects the mobility. This biaxial $\left(\epsilon_{\|}=\epsilon_{x x}=\epsilon_{y y}\right)$ in-plane strain reduces the cubic crystal symmetry of the SiGe layers leading to a splitting of the heavy- and light-hole valence bands ${ }^{15}$ as well as the eventual splitting of conduction band valleys, ${ }^{15}$ with the consequent modification of the effective mass $\left(m_{i}^{*}\right)$ and mobility of the carriers $\left(\mu_{i} \propto 1 / m_{i}^{*}\right)$. Thus, in order to design and fabricate devices with specific transport properties based on strained SiGe layers, a precise knowledge of the alloy composition and in-plane strain is mandatory.

Among the variety of techniques commonly used for strain and composition determination, x-ray diffraction (XRD) and Raman spectroscopy play a major role. However, in some practical situations XRD is not the most convenient technique, e.g., due to the required large crystal lateral size of the samples (typically $l \approx 1 \mathrm{~mm}$ ), or due to the necessity of performing angle-resolved measurements. In contrast, micro-Raman spectroscopy appears as a suitable alternative since it does not impose any of these technical constraints. The typical lateral dimensions of the samples are no longer a limitation due to the small diameter of the focused laser spot ( $\approx 1 \mu \mathrm{m}$ for the visible range). In principle, a single measurement in back-scattering geometry should be enough for a precise determination of the composition of the layers given the in-plane strain, or vice versa. This approach has been already applied by several authors to strained SiGe layers, ${ }^{16-25}$ pointing in their work to the difficulties encountered in some situations to attain the desired accuracy. Although the underlying mathematics employed to extract composition and/or strain values from the measured Raman spectra is extremely simple, a cumbersome interrelation between the involved physical parameters easily leads to misinterpretation of the experimental results. It is instructive to search deeper for the origin of such drawback of the Raman technique. In the 
linear response approximation, the Raman frequencies of the principal alloy vibrational modes (Ge-Ge, $\mathrm{Si}-\mathrm{Ge}$ and $\mathrm{Si}-\mathrm{Si}$ ) for a biaxially strained $\mathrm{SiGe}$ layer read as

$$
\omega^{i}\left(x, \epsilon_{\|}\right)=\omega_{0}^{i}(x)+b_{s}^{i}(x) \times \epsilon_{\|},
$$

where the superindex $i$ accounts for the $\mathrm{Ge}-\mathrm{Ge}, \mathrm{Si}-\mathrm{Ge}$, or $\mathrm{Si}-$ Si vibrational mode, $x$ is the Ge content, $\epsilon_{\|}$is the in-plane strain, $\omega_{0}$ is the phonon frequency for the unstrained case, and $b_{s}(x)$ is the strain shift coefficient. We point out that, strictly speaking, $b_{s}=b_{s}(x, \alpha)$, where $\alpha$ accounts for the strain status of the system, since its actual value is determined by the character of the stress being hydrostatic or anisotropic (uniaxial, biaxial, etc.). As shown previously for lower dimensional structures like quantum dots, ${ }^{22,26} \alpha$ strongly depends on dot shape, wetting layer composition, and cap layer thickness. Nevertheless, in the present case of strictly two-dimensional systems, such a dependence can be neglected due to the pure biaxial nature of the stress exerted on the SiGe layers. According to Eq. (1), $x$ or $\epsilon_{\|}$can be computed by performing a single Raman measurement $(\omega)$ with the previous knowledge of $\omega_{0}$ and $b_{s}$. Whereas, $b_{s}^{i}(x)$ was only recently reported for the whole compositional range by Reparaz et al. $^{23,24}$ and independently by Pezzoli et al., ${ }^{25}$ a very poor agreement is found in the literature for the values of $\omega_{0}^{i}(x)$. It turns out that the discrepancies appear if the samples were grown using different techniques and/or conditions (see, for example, Refs. 16, 18, 24, 25, and 27-29). In consequence, for a given $x$ the determination of $\epsilon_{\|}$using Eq. (1) results highly unreliable. For instance, the use in Eq. (1) of the different values for $\omega_{0}$, reported in Refs. 24 and 29, leads to a discrepancy in the absolute value of $\epsilon_{\|}$as high as $1 \%$, which is unacceptable considering that the maximum inplane strain between $\mathrm{Si}$ and $\mathrm{Ge}$ is $4.2 \%$. This uncertainty in the unstrained phonon frequency $\omega_{0}$ in SiGe alloys would, thus, strongly restrict the applicability of the Raman scattering technique alone to the characterization of strained layers of the pure materials; an unfortunate situation which constitutes the motivation for the present study.

In this work, we investigate the origin of the large scatter of the $\omega_{0}$ values reported by many groups for the unstrained frequency of the optical phonon modes in SiGe alloys. $^{16,18,24,25,27-29}$ Performing a series of sequential annealing steps to several samples containing a strained SiGe layer with different Ge concentrations, we demonstrate that the formation during the growth procedure of Ge richer regions within the alloy largely determines the value of $\omega_{0}$ that would be measured by Raman scattering. The optical phonon frequency of the $\mathrm{Ge}-\mathrm{Ge}$ and $\mathrm{Si}-\mathrm{Si}$ alloy modes appears to be sensitive to the local composition of the corresponding atomic species. The latter obviously depends on the degree of homogeneity of the sample concerning alloy disorder. Furthermore, we propose a simple analytical/graphical method for the determination of average composition and strain in SiGe alloys out of the data from a single Raman measurement. Since the rational of the proposed method takes into account compositional inhomogeneity effects, we are able to provide a set of general criteria that allows for the successful processing of the Raman data in almost any experimental situation, irrespective of the degree of alloy disorder and level of strain relaxation of the alloy layers.

\section{EXPERIMENTAL DETAILS}

The samples were grown on (001)-oriented Si substrates using a solid-source molecular beam epitaxy (MBE) equipment. Initially, the Si substrates were heated to $900{ }^{\circ} \mathrm{C}$ for $\mathrm{SiO}_{2}$ desorption, after which a $100 \mathrm{~nm}$ thick Si buffer layer was deposited using a decreasing substrate temperature ramp between 900 and $500^{\circ} \mathrm{C}$. In the following stage, $\mathrm{Si}_{1-x} \mathrm{Ge}_{x}$ layers were grown at $400^{\circ} \mathrm{C}$ (substrate temperature) with various $x$ values ranging between 0 and 0.5 and with thicknesses between 8 and $700 \mathrm{~nm}$. A list of all the grown samples is shown in Table I. The as-grown samples were removed from the MBE chamber for further characterization using Raman spectroscopy, XRD, and spectroscopic optical ellipsometry. Subsequently, each sample was re-introduced in the MBE chamber in order to perform cumulative thermal annealing in successive steps of $50{ }^{\circ} \mathrm{C}$ between 450 and $750{ }^{\circ} \mathrm{C}$. After each annealing step the samples were removed from the chamber for structural and optical inspection. The total number of investigated samples, considering each annealing step, amounts to 48 (6 different concentrations $\times 8$ annealing temperatures). We point out that each annealing step was performed in the MBE chamber to avoid any oxidation of the samples due to the low residual pressure of the chamber (about $10^{-10}$ mbar). Several initial annealing attempts performed in a furnace with Ar atmosphere had led, despite previous purging of the setup, to strong surface oxidation of the SiGe layers.

Raman spectra were recorded using a LabRam HR800 system in backscattering geometry with a spectral resolution of about $0.3 \mathrm{~cm}^{-1}$. As excitation source we used the $514.5 \mathrm{~nm}$ line of an air-cooled $\mathrm{Ar}^{+}$ion laser, which was focused on the sample to a spot of around $2 \mu \mathrm{m}$ in diameter. Raman measurements were performed in crossed linear polarization configuration $\mathrm{z}(\mathrm{x}, \mathrm{y}) \bar{z}$, in order to minimize the contribution of the second-order Raman signal (Si 2TA) arising from the Si substrate, whose energy is close to that of the Ge-Ge mode. XRD data for the symmetric reflection (004) were collected using a Siemens D-5000 diffractometer. Xray reciprocal space maps (q-plots) around two asymmetric reflections: (224) and $(2 \overline{2} 4)$ were measured for the as-grown samples and some of the annealed ones using a Bruker D8 with a two dimensional multi-channel detector. Note that the

TABLE I. $\mathrm{Si}_{1-x} \mathrm{Ge}_{x}$ alloy-layer thickness $(d)$ of the different samples determined by spectral ellipsometry. Ge content for the as-grown layers obtained from XRD measurements $\left(x_{x r d}\right)$ and using the model discussed in Sec. IV B $\left(x_{\text {model }}\right)$. Numbers in parentheses represent error bars.

\begin{tabular}{llll}
\hline \hline Sample & $d(\mathrm{~nm})$ & $x_{x r d}$ & $x_{\text {model }}$ \\
\hline A & $8(1)$ & $0.15(1)$ & $0.11(1)$ \\
B & $700(10)$ & $0.18(1)$ & $0.18(1)$ \\
C & $570(5)$ & $0.22(1)$ & $0.24(1)$ \\
D & $114(4)$ & $0.40(1)$ & $0.41(2)$ \\
E & $43(2)$ & $0.44(1)$ & $0.48(2)$ \\
F & $20(1)$ & $0.47(1)$ & $0.47(2)$ \\
\hline
\end{tabular}


asymmetric reflections allow for the determination of the lattice parameter in both the in-plane and out-of-plane directions. Spectroscopic ellipsometry measurements in the range from 1.4 to $5.2 \mathrm{eV}$ were performed at room temperature using a SOPRA ES4G ellipsometer. As excitation light source we used a $75 \mathrm{~W}$ high-pressure Xe arc lamp and the reflected light was coupled into a double prism/grating monochromator of $750 \mathrm{~mm}$ equivalent focal length, and detected with a multi-alkali photomultiplier tube. The ellipsometry spectra of all measured alloy layers was used to estimate the surface roughness of the layers through the decrease of the imaginary part of the dielectric function $\left(\epsilon_{2}\right)$ at the energy of the interband optical transition denoted as $\mathrm{E}_{2} \cdot{ }^{30} \mathrm{~A}$ surface roughness of about $1 \mathrm{~nm}$ was detected in the worst case, which implies that the alloys exhibit practically flat surfaces.

\section{THEORETICAL CONSIDERATIONS}

The application of a stress to a solid leads to a deformation of its crystal structure, which in the linear approximation is usually expressed as ${ }^{31} \sigma_{i j}=c_{i j k l} \times \epsilon_{k l}$, where $\sigma_{i j}$ and $\epsilon_{k l}$ are the stress and strain tensor, respectively, and $c_{i j k l}$ is the fourth-rank tensor corresponding to the elastic constants. The high degree of symmetry of cubic crystals reduces the number of independent components of $c_{i j k l}$ to only three, which expressed in the more comfortable $6 \times 6$ matricial form $^{31}$ are denoted by $C_{11}, C_{12}$, and $C_{44}$. In the particular case of a biaxially stressed layer in the x,y plane, it holds $\sigma_{x x}=\sigma_{y y}=\sigma_{\|}$and $\sigma_{z z}=\sigma_{\perp}=0$. By replacing in the stress/ strain equation we obtain the following relations:

$$
\begin{gathered}
\sigma_{\|}=\left(C_{11}+C_{12}\right) \times \epsilon_{\|}+C_{12} \times \epsilon_{\perp}, \\
\epsilon_{\perp}=-\frac{2 C_{12}}{C_{11}} \times \epsilon_{\|} .
\end{gathered}
$$

On the other hand, Eq. (2) expresses how the stress translates into internal strain, Eq. (3) stands for the Poisson effect: An in-plane compression/expansion leads to an expansion/compression in the growth direction. In addition, the in-plane strain in the SiGe layers is expressed as $\epsilon_{\|}=\left(a_{\|}-a_{S i G e}\right)$ $/ a_{S i G e}$, where $a_{S i G e}$ is the lattice constant of the SiGe alloy for the unstrained case $\left(\epsilon_{i j}=0 \forall i, j\right)$. Thus, combining the previous relation for $\epsilon_{\|}$with Eqs. (1) and (3), we obtain a system of two equations, which can be directly related to measurable quantities such as $\omega^{i}$ (obtained from Raman spectra), $a_{\|}$and $a_{\perp}$ (both from XRD measurements) given by

$$
\begin{gathered}
\omega^{i}\left(x, \epsilon_{\|}\right)=\omega_{0}^{i}(x)+b_{s}^{i}(x) \times\left[\frac{a_{\|}-a_{S i G e}(x)}{a_{S i G e}(x)}\right], \\
{\left[\frac{a_{\perp}-a_{S i G e}(x)}{a_{S i G e}(x)}\right]=-\frac{2 C_{12}(x)}{C_{11}(x)} \times\left[\frac{a_{\|}-a_{S i G e}(x)}{a_{S i G e}(x)}\right] .}
\end{gathered}
$$

The values used here for $b_{s}(x), a_{S i G e}(x)$, and $C_{i j}(x)$ are extracted from Refs. 24, 32, and 33, respectively, and listed in Table II. Actually, the determination of $x$ and $\epsilon_{\|}$can be fully achieved from XRD data, using Eq. (5) which depends on $a_{\|}$and $a_{\perp}$ only. Nevertheless, as already anticipated, it is highly desirable to achieve this only by performing Raman measurements due to the versatility of this technique. The main problem with Eq. (4) is the large scatter in the $\omega_{0}^{i}$ values, which leads to a large error in the determination of $x$ or $\epsilon_{\|}$. To overcome this problem, we need to understand the reason for the different reported values of $\omega_{0}^{i}$, which will naturally lead us to a consistent picture of general validity for the interpretation of the Raman spectra of SiGe alloys. For the sake of clarity, we present first the experimental results and then we explain the observed phenomenology.

\section{RESULTS AND DISCUSSION}

\section{A. Origin of the scatter of the $\omega_{0}$ values}

In this section, we intend to clarify the origin of the large scatter reported in the literature for $\omega_{0}$ (see Refs. 16, 18, 24, 25, and 27-29). For this purpose, we have performed a series of cumulative sequential annealing steps in samples with different $x$ between 0 and 0.5. In Fig. 1(a), we show representative spectra of the as-grown samples. The strongest peak observed in each spectrum at $520.7 \mathrm{~cm}^{-1}$ arises from the Si substrate first-order longitudinal-optical (LO) phonon. This peak has been cut off in intensity for better visualization of the remaining modes. At lower frequencies three modes become apparent, which are identified as the principal alloy modes as follows: (i) $\omega^{G e G e} \approx 300 \mathrm{~cm}^{-1}$, (ii) $\omega^{\text {SiGe }}$

TABLE II. Composition dependence of the strain shift coefficients for the Ge-Ge, Si-Ge, and Si-Si modes extracted from Ref. 24. Lattice constants and elastic constants extracted from Refs. 32 and 33, respectively. The polynomial parameters for the unstrained frequencies were obtained by a combined fit to the data of Refs. 28 and 34 .

Strain shift coefficients $\left(\mathrm{cm}^{-1}\right)$

Lattice constant $(\AA)$

Elastic constants (GPa)

Unstrained phonon frequencies $\left(\mathrm{cm}^{-1}\right)$

$$
\begin{aligned}
& b_{s}^{G e G e}(x)=-190 \times(x-1)^{4}-460 \\
& b_{s}^{S i G e}(x)=-190 \times(x-1)^{4}-555 \\
& b_{s}^{S i S i}(x)=-190 \times(x-1)^{4}-650 \\
& a_{S i G e}=5.431+0.198 \times x+0.028 \times x^{2} \\
& C_{11}(x)=165.77-37.24 \times x \\
& C_{12}(x)=63.93-15.67 \times x \\
& \omega^{G e G e}=284+5 \times x+12 \times x^{2} \\
& \omega^{S i G e}=400+29 \times x-95 \times x^{2}+213 \times x^{3}-170 \times x^{4} \\
& \omega^{S i S i}=520.7-68 \times x
\end{aligned}
$$




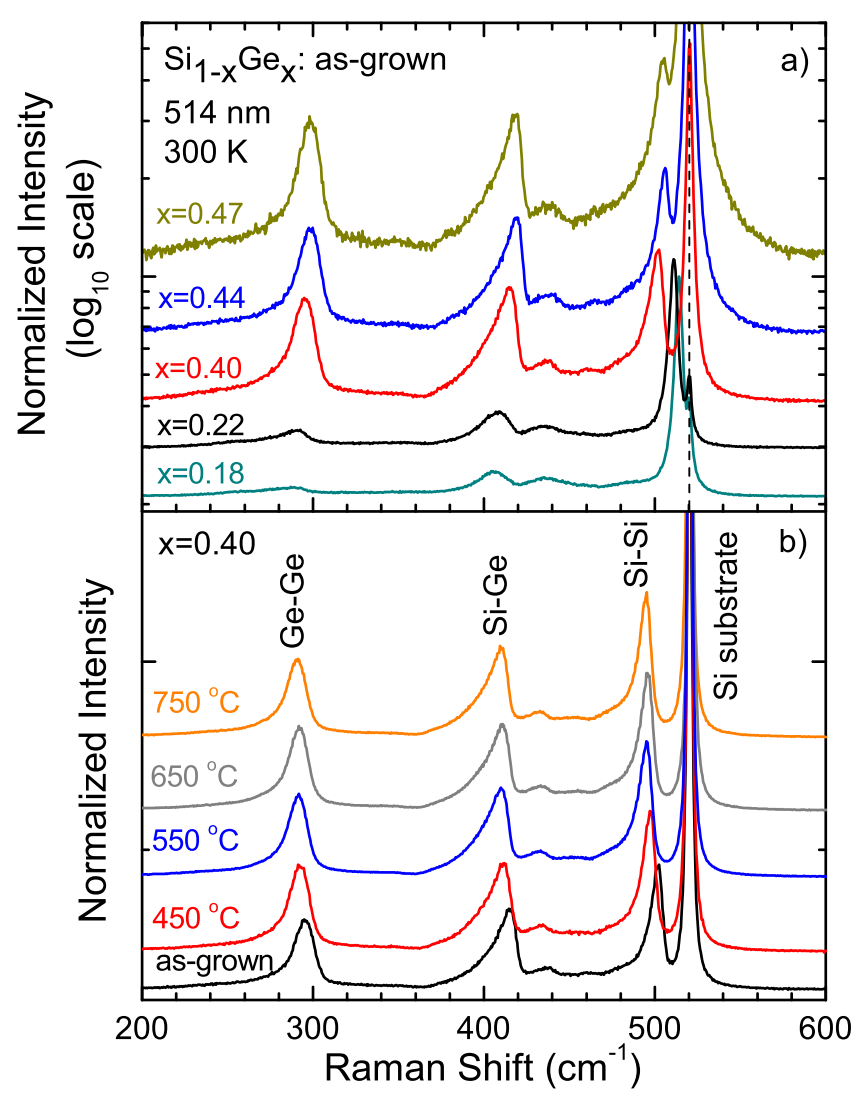

FIG. 1. (a) Representative Raman spectra of the $\mathrm{Si}_{1-x} \mathrm{Ge}_{x}$ as-grown samples with $x=0.18,0.22,0.40,0.44,0.47$ and the Raman intensity plotted in logarithmic scale. (b) Representative spectra of the as-grown sample with $x=0.40$ and for different annealing temperatures. Peak assignment to the alloy optical Raman modes is indicated.

$\approx 410 \mathrm{~cm}^{-1}$, and (iii) $\omega^{S i S i} \approx 510 \mathrm{~cm}^{-1}$. In all cases, the peak intensities have been normalized to that of the LO mode from the Si substrate. The phonon frequency of each alloy mode for different Ge contents was obtained from the maximum position of asymmetric Gaussian line-shapes fitted to the Raman spectra. In particular, the Si-Ge mode exhibits a large asymmetry for the higher Ge compositional range which originates from alloy disorder. We anticipate that the Raman spectrum of each sample is highly sensitive to variations in the local composition (at the nanoscale) of the alloy layers. Alloys of materials with very dissimilar masses like $\mathrm{Si}$ and $\mathrm{Ge}$ exhibit peculiar vibrational properties which, to the lowest order approximation are roughly described by the so-called one-bond-one-mode model. ${ }^{35}$ Within this model, vibrations are highly localized to single bonds, being the main phonon modes of the alloy the ones associated to the three kinds of existing atom pairs $\mathrm{Si}-\mathrm{Si}, \mathrm{Si}-\mathrm{Ge}$, or Ge-Ge. The vibrational frequency of each alloy mode, thus, is very sensitive to the atomic composition of its immediate environment. Even more important is the fact that the main contribution to the Raman signal stemming from the Ge-Ge and $\mathrm{Si}-\mathrm{Si}$ modes arises from the $\mathrm{Ge}$ and $\mathrm{Si}$-rich regions, respectively. Hence, in inhomogeneous samples exhibiting certain degree of compositional inhomogeneity, the different alloy modes would sense a different average composition and residual strain, the latter in case of a strained alloy layer. In fact, with increasing $\mathrm{Ge}$ content the Ge-Ge and $\mathrm{Si}-\mathrm{Ge}$ modes shift to higher frequencies, whereas the $\mathrm{Si}-\mathrm{Si}$ mode displays initially a redshift and then a blueshift. Since the alloy layers are all grown epitaxially on $\mathrm{Si}$, the biaxial in-plane strain also increases with Ge content, causing an overall blueshift of the phonon modes. The main issue of this work is to obtain a reliable procedure to separate the contributions to the mode frequency given by Eq. (1) arising from the $\omega_{0}$ and $\epsilon_{\|}$terms, which are mixed in the frequency shifts observed in the spectra of Fig. 1.

Figure 1(b) shows the effect of thermal annealing on the phonon frequencies for a representative sample with $x=0.40$. As the annealing temperature increases all the phonon frequencies shift systematically to lower energies. Two main candidates appear as responsible for the observed shifts: Interdiffusion of $\mathrm{Si}$ from the substrate into the alloy layer ${ }^{36}$ and the combined effect of alloy homogenization by Ge redistribution and strain relaxation within the layers. If interdiffusion occurs, its effect would be to decrease the average Ge content of the layers, which would affect in opposite directions the frequencies of the Ge-Ge and $\mathrm{Si}-\mathrm{Si}$ modes (see Fig. 3 ), contrary to what is observed in the Raman spectra of the annealed samples. Moreover, we have grown three samples (B, C, and D) with thicknesses large enough to ensure that if interdiffusion with the substrate takes place, its effect is negligible for any practical means. The behavior of the Raman frequencies upon annealing is, in fact, exactly the same for the thick and thin alloy layers, what completely rules out interdiffusion as the possible cause of the observed frequency shifts.

The dependence of the out-of-plane lattice parameter $a_{\perp}(x)$ on annealing temperature is shown in Fig. 2 for all samples. We have confirmed first that for all compositions the as-grown samples are pseudomorphic to the Si substrate by performing X-ray reciprocal space maps (not shown). Figure 2 demonstrates that with increasing annealing temperature $a_{\perp}$ decreases ( $a_{\|}$increases) leading to smaller values of $\epsilon_{\|}$, which, in turn, would result in smaller frequencies for all the modes with rates given by $b_{s}^{i}$ (see Eq. (4)). This effect is more pronounced for the thicker samples (B, C, and D),

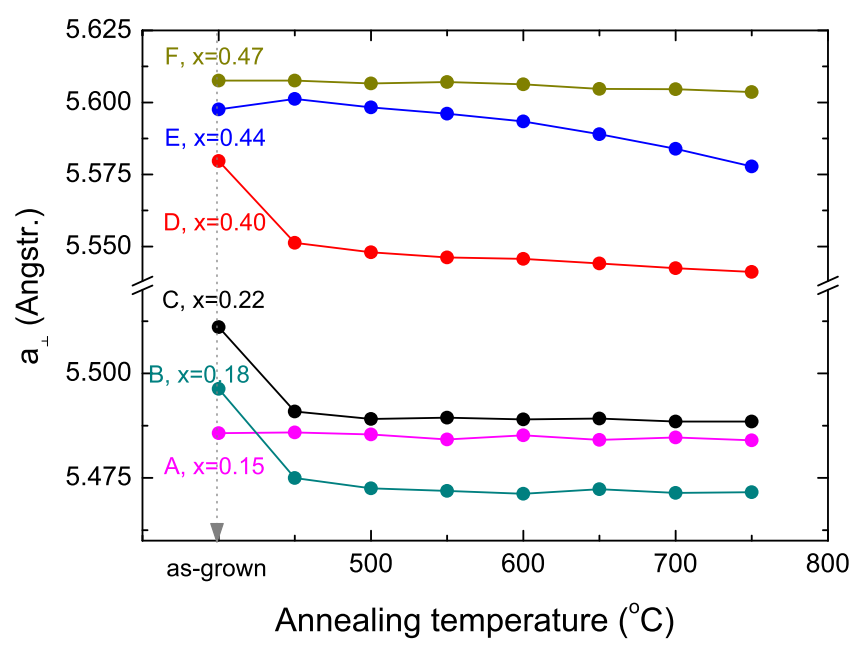

FIG. 2. Out-of-plane lattice parameter $\left(a_{\perp}\right)$ of the SiGe alloy layers determined by XRD measurements as a function of the annealing temperature for samples A to E. The vertical line corresponds to the as-grown samples. Error bars of the data points are around $\pm 0.001 \AA$. 


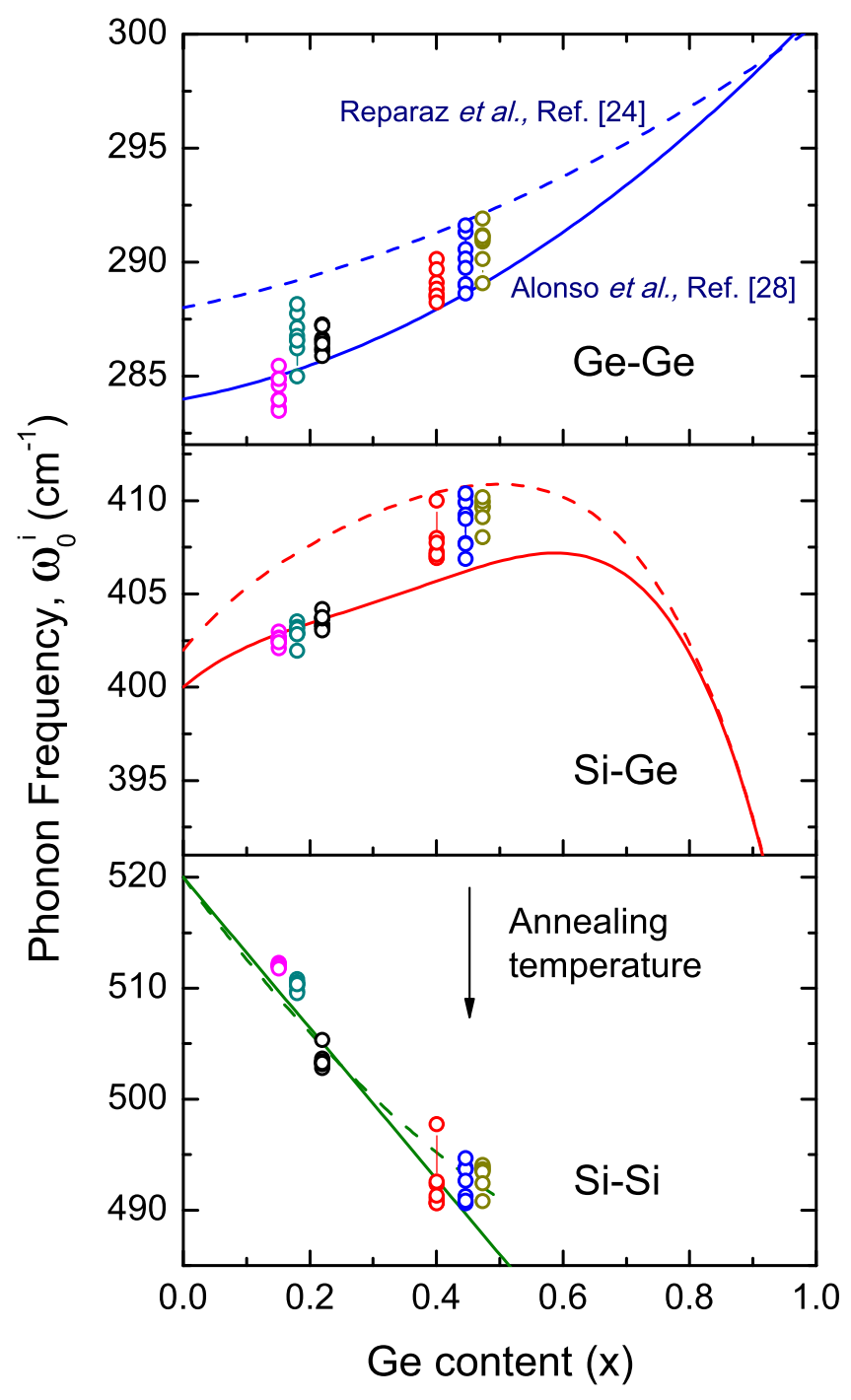

FIG. 3. Computed values for $\omega_{0}^{i}$ using Eqs. (4) and (5) for each vibrational mode and annealing temperature. The dashed and solid curves represent the values of $\omega_{0}^{i}$ from Refs. 24 and 28, respectively. Error bars of the data points (not shown since they are smaller than the symbol size) are around $\pm 0.5 \mathrm{~cm}^{-1}$

indicating almost full strain relaxation after the first annealing step. For the thinner samples (A, E, and F) strain relaxation is less pronounced, which is easily explained considering that the total elastic energy due to the lattice mismatch with the Si substrate scales linearly with the thickness of the layers. The most important point here is that all these strain relaxation effects which differ from sample to sample, depending mainly on thickness, are not able solely to account for the magnitude of the observed monotonic redshifts of the mode frequencies with increasing annealing temperature. Incidentally, we point out that Fig. 2 constitutes itself an important result since it shows that over a certain thickness, the strained SiGe layers are largely strain relaxed at high annealing temperatures. This might be important for a correct design of devices based on strained SiGe layers which are intended to operate above room temperature.

Thus, having ruled out interdiffusion and strain relaxation effects alone, we are led to the conclusion that a different degree of compositional disorder is at the origin of the sample dependence of $\omega_{0}$. Taking advantage of the fact that the different samples were characterized by XRD and of our knowledge of the strain shift coefficients, ${ }^{24}$ we are able to reverse the problem and compute the value of $\omega_{0}$ for each studied sample. According to Eqs. (4) and (5), this problem can be solved exactly using the measured values for $\omega^{i}$ and $a_{\|}$. For the as-grown samples this calculation is straightforward since the SiGe layers are pseudomorphic to the substrate $\left(a_{\|}=a_{S i}\right)$. As the annealing temperature increases, the XRD results indicate that the strain within the layers gradually relaxes $\left(a_{\|} \neq a_{S i}\right)$. In Fig. 3, we show the computed values of $\omega_{0}$ for each phonon mode and annealing temperature. In addition, we have included the reported values from Refs. 24 and 28, which represent two limiting cases for the present set of samples as far as compositional inhomogeneities are concerned. The values reported in Ref. 24 were measured in fully strained samples grown by $\mathrm{MBE}$ at $400^{\circ} \mathrm{C}$. Since $\mathrm{MBE}$ is a non-equilibrium growth technique, it is known that due to the low deposition temperature, Ge tends to aggregate during the growth of the strained alloy layer. An inhomogeneous strain driven decomposition of the alloy leads to a nonrandom distribution of the $\mathrm{Ge}$ atoms at the nanoscale, ${ }^{37}$ which affects the measured values for $\omega_{0}$. In contrast, the $\omega_{0}$ values reported in Ref. 28 were obtained from samples grown using liquid-phase epitaxy (LPE) which is an equilibrium growth technique and, consequently, the Ge distribution is expected to be as random or homogeneous as possible. The open symbols in Fig. 3 show how the computed values of $\omega_{0}$ gradually shift down from the curve extracted from Ref. 24 towards that of Ref. 28. The observed behavior represents the experimental confirmation of our ansatz that, despite the fact that the average alloy composition remains essentially constant as revealed by XRD, the distribution of Ge atoms within the layers progressively randomizes with each annealing step, leading to changes in phonon frequencies associated to the thermally induced compositional homogenization of the alloy.

\section{B. Determination of Ge content and residual strain through a single Raman measurement}

We now describe the procedure by which both $x$ and $\epsilon_{\|}$ can be accurately determined for any strained SiGe layer. For that purpose we employ the measured Raman frequencies of the Ge-Ge and Si-Si modes, having the values of $\omega_{0}$ extracted essentially from Ref. 28 (see Table II) as a reference for a random distribution of the Ge atoms within the alloy layer. The Si-Ge mode, in contrast, is not relevant for such a determination due to its approximately flat dependence in the mid-compositional range (see Fig. 3), which makes this mode almost insensitive to composition and/or strain changes. Nevertheless, for Ge contents above 0.6, where the Si-Si mode is usually absent in the Raman spectra of the alloy, the Si-Ge mode can be used instead (fortunately, for $0.6<x<1$ the Si-Ge mode exhibits a marked composition dependence). We demonstrate the procedure choosing as an example the as-grown sample $\mathrm{E}\left(x_{x r d}=0.44\right)$, which exhibits following Raman frequencies: $\omega^{G e G e}=299.4 \mathrm{~cm}^{-1}$ and $\omega^{S i S i}=505.7 \mathrm{~cm}^{-1}$. Applying Eq. (4) independently for 
each mode we obtain $x_{G e G e}=0.54$ and $x_{S i S i}=0.35$, which both clearly differ from the average composition $x_{x r d}=0.44$ determined by XRD. We note that whereas XRD probes a comparatively large volume of the sample, yielding the mean Ge content of the layers $(x)$, the Raman modes are highly sensitive to the local environment of the vibrating atoms mainly circumscribed to the first-nearest neighbor sphere. Thus, the formation of compositional inhomogeneities is only observed with Raman scattering, but remains unnoticed in the XRD spectra. This effect has been already theoretically studied, ${ }^{38,39}$ though no detailed experimental investigation is available yet to the best of our knowledge. In consequence, we have to modify Eq. (4) in order to include the compositional inhomogeneity effect as follows:

$$
\begin{gathered}
\omega^{G e G e}\left(x, \epsilon_{\|}\right)=\omega_{0}^{G e G e}(x+m \times R)+b_{s}^{i}(x) \times \epsilon_{\|}, \\
\omega^{S i S i}\left(x, \epsilon_{\|}\right)=\omega_{0}^{S i S i}(x-n \times R)+b_{s}^{i}(x) \times \epsilon_{\|},
\end{gathered}
$$

where $R$ is a correction factor to the average composition $(x)$, which accounts for the degree of compositional disorder within the alloy layer. The factors $m=1-x$ and $n=x$, in addition, allow for the local composition unbalance sensed by each mode. We remark that no correction ${ }^{39,40}$ is applied to the strain term of Eq. (4), which is the usual macroscopic averaged $\epsilon_{\|}$as probed by XRD measurements. As seen in the example of sample $\mathrm{E}$, the $\mathrm{Ge}-\mathrm{Ge}$ mode always probes a higher Ge content $\left(x_{G e G e} \geq x_{x r d}\right)$ but the Si-Si mode a lower one $\left(x_{S i S i} \leq x_{x r d}\right)$. Although $m$ and $n$ are expressed as linear functions of $x$ (simply satisfying the continuity relation $m+n=1$ ), more sophisticated models with higher order terms are possible. Nevertheless, attempts using second order terms did not bring any real benefit. The procedure consists in solving the system of two coupled equations (6) and (7) with two unknowns $x$ and $R$ for all the as-grown samples, for which certainly holds that $a_{\|}=a_{S i}$. We list the obtained layer compositions in Table I labeled as $x_{\text {model }}$, together with the values obtained from XRD measurements $x_{x r d}$. The agreement between these two determinations is more than satisfactory, which demonstrates the validity of the developed method.

We go a step further and relax the condition of pseudomorphism previously imposed, which holds true for the asgrown samples but not for the general case. Now is $a_{\|} \neq a_{S i}$ which applies to most of the annealed samples (see Fig. 2), since the effect of the successive annealing steps is not only to randomize the Ge distribution but also to relax (partially or totally) the built-in strain due to the lattice mismatch to the $\mathrm{Si}$ substrate. This relaxation condition can be easily expressed by introducing in the strain term of Eq. (4) a strain factor $S$ defined as $\epsilon_{\|}=S \times \epsilon_{i s o}(x)=S \times\left[a_{\text {SiGe }}(x)-a_{S i}\right]$ $/ a_{S i G e}(x)$. We note that by definition, $S=0$ represents the fully relaxed case, while $S=1$ corresponds to the fully strained case. With this modification, Eqs. (6) and (7) read as

$$
\begin{aligned}
\omega^{G e G e}\left(x, \epsilon_{\|}\right) & =\omega_{0}^{G e G e}(x+m \times R)+b_{s}^{i}(x) \times S \times \epsilon_{i s o}(x), \\
\omega^{S i S i}\left(x, \epsilon_{\|}\right) & =\omega_{0}^{S i S i}(x-n \times R)+b_{s}^{i}(x) \times S \times \epsilon_{i s o}(x) .
\end{aligned}
$$

We now face the problem of solving a system of two coupled equations with $x, R$, and $S$ as unknowns. Although the present problem is not uniquely solvable for obvious reasons, it is still possible to guess a practical solution by a graphical procedure. For this purpose, we return to the previous example of sample E, where we now aim at finding a graphical solution for Eqs. (8) and (9). In Fig. 4(a), we show the three-dimensional (3D) plots of the strain parameter $S=S(x, R)$ constituting possible solutions of each of those equations. The left surface (orange) represents the solutions for the $\mathrm{Si}-\mathrm{Si}$ mode, and the right one (violet) relates to the Ge-Ge mode. The intersection between these surfaces gives the physically possible solutions of the problem, in which both strain factors $(S)$ from Eqs. (8) and (9) are equal, i.e., both phonon modes are sensing the same amount of strain relaxation, if any. This intersection curve corresponds to an in-plane strain ranging from 0 to $\epsilon_{\|}^{\max }$ $=\left[a_{S i G e}(0.44)-a_{S i}\right] / a_{S i G e}(0.44)$. It becomes clear from Fig. 4(a) that the Ge content cannot be directly deduced without making further assumptions on the strain within the alloy layer. This is due to the high Ge content of $x=0.44$, which introduces a large strain for pseudomorphic growth on $\mathrm{Si}$, thus, leading to an extended intersection curve which spans an

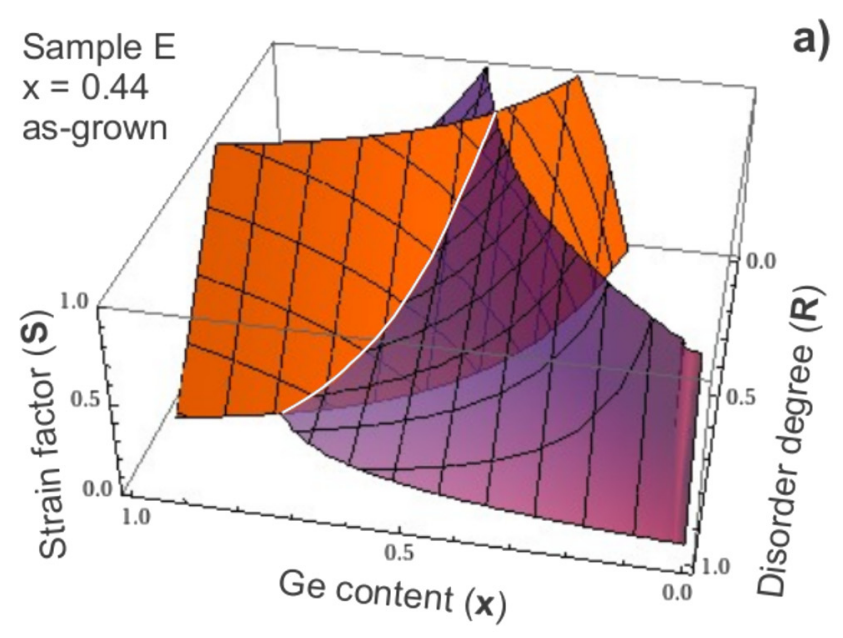

$750^{\circ} \mathrm{C}$ annealed

b)

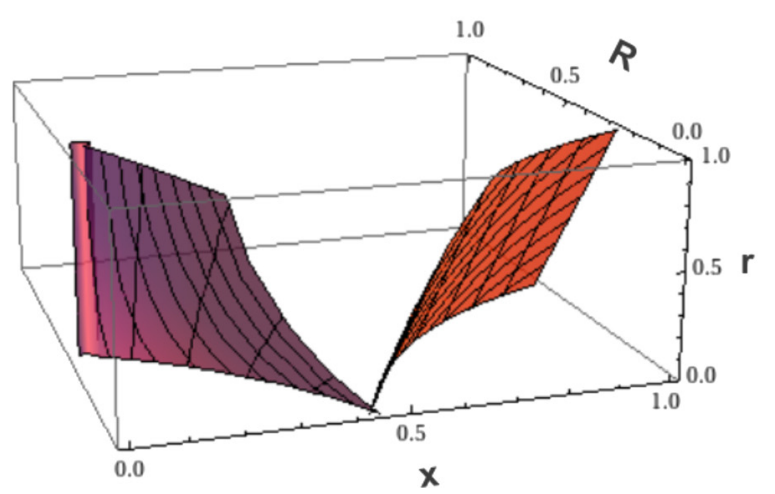

FIG. 4. Three dimensional plots showing the solutions of Eqs. (8) and (9) for the Ge-Ge (violet) and Si-Si (orange) modes, respectively. In panel (a), we show the two surfaces for the as-grown sample E. The same surfaces are shown in (b) for sample E after all the annealing sequences. The physically reasonable solutions are given by the intersection of the two dimensional surfaces as marked with a white line. 
interval of $x$ and $R$ values. Here we make use of what we certainly know from XRD data that the as-grown alloy layers are almost fully strained $(S \approx 1)$. Closer inspection of similar $3 \mathrm{D}$ plots obtained for all other as-grown samples indicate that this condition is always fulfilled for solutions for which the inhomogeneity factors $R$ are minimal.

We consider next the case of sample $\mathrm{E}$ after all the annealing steps $\left(750^{\circ} \mathrm{C}\right)$. In Fig. 4(b), we show again the strain factors $S=S(x, R)$ from Eqs. (8) and (9) plotted together but computed independently. We note that the only difference with the previous case is the measured values for $\omega^{G e G e}$ and $\omega^{S i S i}$. The two surfaces exhibit a completely different behavior, being the solution only restricted to almost a single vector in the $x-R-S$ space. In this particular case, it corresponds to the point $(x, R, S)=(0.43,0.01,0.05)$, in good agreement with the composition determined by XRD $x_{x r d}=0.44$ and the strain value $\epsilon \approx 0$ of this relaxed sample. Consequently, the correction factor $R$ results almost zero, reflecting the fact that after repeated annealing the distribution of the Ge atoms in the alloy becomes random (see also Fig. 3, where the data points in this case fall on top of the reference curve of Ref. 28). The 3D plots given in Fig. 4 show that whereas strained layers exhibit a continuous set of solutions over a relatively large compositional range, partially (totally) relaxed layers show a set of solutions with a small compositional dispersion.

Finally, we show in Fig. 5 the projection onto the $x, S$ plane of the intersection curves obtained by the procedure demonstrated in Fig. 4 as solutions of the system given by Eqs. (8) and (9) for all the as-grown samples (solid lines) and the fully annealed ones (lines inside circular marks). The dotted lines represent contour plots for different values of $R$, though calculated only for the as-grown samples. While the solutions for strained samples exhibit an interval of compositions, the relaxed layers converge to almost a unique solu-

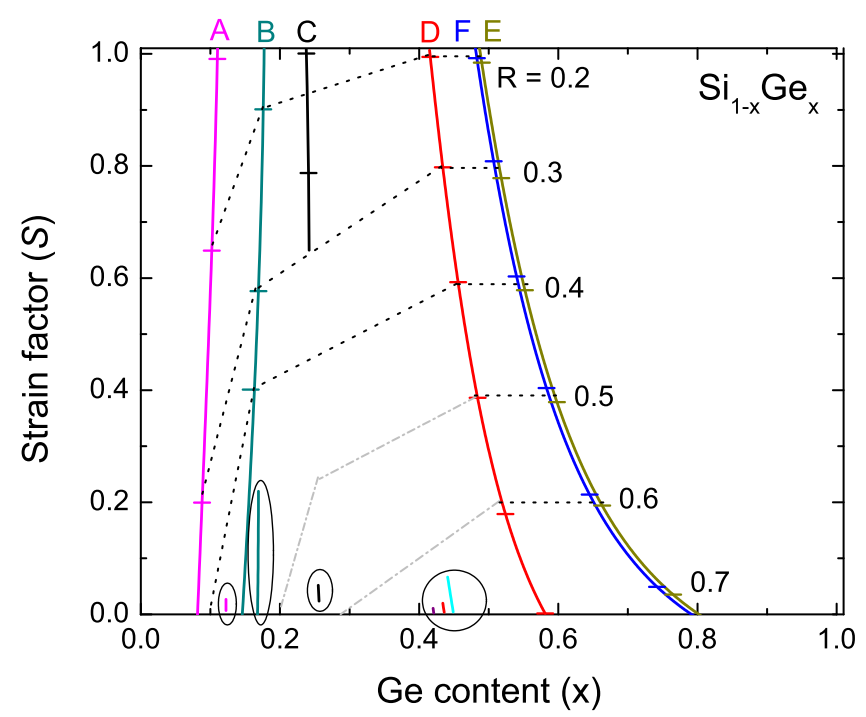

FIG. 5. Solutions of Eqs. (8) and (9) for all the as-grown samples (solid lines) and annealed ones (circled lines) represented in the $S, x$ plane. The dotted black lines connect points with constant values of compositional inhomogeneity degree $R$, representing real solutions, whereas the grey dotted lines are a guide to the eye, representing estimated solutions. tion. In the latter case, we estimate from Fig. 5 that the accuracy in the determination of the Ge content is about \pm 0.01 . For the strained samples, an extra criterion must be adopted due to extra variability introduced by the in-plane strain present in the alloy layers. Although all $(x, S)$ points on the curves plotted in Fig. 5 are mathematical solutions of the problem, only certain $(x, R, S)$ are physically realistic. For fully strained layers $(S=1)$ the right solution minimizes the inhomogeneity factor $R$. In contrast, for unknown strain relaxation and alloy disorder degree we found out empirically that the best result corresponds to the triad which maximizes the product $S \cdot R$. From an exhaustive analysis of all the 48 investigated samples we have found that the upper limits for the error in the determination of the $\mathrm{Ge}$ content rises to \pm 0.02 for $x<0.3$ and \pm 0.03 for $x>0.3$. We note that large values of $R$ are possible in highly inhomogeneous samples which, nevertheless, are not usually obtained with the conventional epitaxial growth techniques.

In practice, it is possible to estimate $x$ and $\epsilon_{\|}$using the following procedure:

1. Measure the Raman spectrum of a given sample and extract $\omega^{G e G e}, \omega^{S i S i}$ and/or $\omega^{S i G e}$.

2. Using Eqs. (8) and (9), construct both surfaces in the $x-R-S$ space in a similar way to the plots of Fig. 4 and inspect the intersection curve given by the constrain $S^{G e G e}(x, R)$ $=S^{S i S i}(x, R)$ (for $x \geq 0.6$ use the SiGe mode instead of the SiSi one).

3. Infer the Ge content and residual strain by analyzing the extension of the intersection curve: If it clasps a whole interval of strain factors containing $S \simeq 1$, then the alloy is under large biaxial strain and the most likely solution is obtained by minimizing the compositional inhomogeneity parameter $R$. Otherwise, search for the solution which maximizes the product $S \cdot R$. Finally, for almost strainrelaxed samples the intersection curve restricts itself to a short segment around values of $S$ close to zero and the solution is obtained with good accuracy directly from the graph.

\section{SUMMARY}

In conclusion, our findings have provided deeper insight into the vibrational properties of semiconductor alloys, which turned out to be crucial for the quantitative understanding of their Raman spectra. In particular, we have found that a different degree of compositional inhomogeneity is the origin of the large scatter of the values reported for the unstrained frequency $\omega_{0}$ of the vibrational modes in SiGe alloys measured by Raman scattering. We have shown that this effect is particularly important in samples grown using non-equilibrium, epitaxial methods like MBE, whereas it is almost absent for growth techniques such as LPE, where the alloy crystallizes onto the substrate following an equilibrium phase diagram. On this basis, we conclude that for fully random alloys $\omega_{0}$ can be reliably obtained from the results published by Alonso and Winer $^{28}$ and summarized in Table II. Additionally, we have developed a simple model that explains the behavior of the alloy phonon modes, valid for samples exhibiting any 
degree of compositional inhomogeneity and strain status. Within this model, we introduce a correction to the Ge content in the form $x \rightarrow(x+m \times R)$ for the Ge-Ge mode and $x \rightarrow(x-n \times R)$ for the $\mathrm{Si}$-Si mode to simultaneously fulfill the equations for the phonon mode frequency $\omega^{G e G e}=$ $\omega\left(x+m \times R, \epsilon_{\|}\right)$and $\omega^{S i S i}=\omega\left(x-n \times R, \epsilon_{\|}\right)$, as given in Ref. 28. This system can be solved exactly if $\epsilon_{\|}$is known a priori, but requires an approximate graphical solution for situations where $\epsilon_{\|}$is unknown. In this way, we have successfully revisited the problem of the experimental determination of alloy composition and residual strain by performing a single Raman measurement. In practice, our analytical/graphical method should be extremely useful in situations where XRD measurements cannot be conducted, repositioning the Raman spectroscopy technique among the top characterization tools in semiconductor nano/micro-technology.

\section{ACKNOWLEDGMENTS}

This work was supported by Spanish Ministry of Science and Innovation (MICINN) through Grants MAT200909480 (PIEZOHM) co-financed by FEDER funds and CSD2010-00044 (Consolider NANOTHERM). I.C.M. is grateful to MICINN for a FPI fellowship. Raman measurements were performed at the Nanotechnology Laboratory of MATGAS Research Center.

${ }^{1}$ F. Schäffler, Semicond. Sci. Technol. 12, 1515 (1997).

${ }^{2}$ M. V. Fischetti and S. E. Laux, J. Appl. Phys. 80, 2234 (1996).

${ }^{3}$ M. Kondo, K. Oda, E. Ohue, H. Shimamoto, M. Tanabe, T. Onai, and K. Washio, IEEE Trans. Electron Devices 45, 1287 (1998).

${ }^{4}$ A. Gruhle, IEEE Trans. Electron Devices 41, 198 (1994).

${ }^{5}$ R. Krithivasan, G. Niu, J. D. Cressler, S. M. Currie, K. E. Fritz, R. A. Reed, P. W. Marshall, P. A. Riggs, B. A. Randall, and B. Gilbert, IEEE Trans. Nucl. Sci. 50, 2126 (2003).

${ }^{6}$ S. Takagi, T. Irisawa, T. Tezuka, T. Numata, S. Nakaharai, N. Hirashita, Y. Moriyama, K. Usuda, E. Toyoda, S. Dissanayake, M. Shichijo, R. Nakane, S. Sugahara, M. Takenaka, and N. Sugiyama, IEEE Trans. Electron Devices 55, 21 (2008).

${ }^{7}$ J. D. Cressler, IEEE Trans. Microwave Theory Tech. 46, 572 (1998).

${ }^{8}$ D. L. Harame, J. H. Comfort, J. D. Cressler, E. F. Crabbé, J. Y.-C. Sun, B. S. Meyerson, and T. Tice, IEEE Trans. Electron Devices 42, 455 (1995).

${ }^{9}$ A. Gruhle, H. Kibbel, U. König, U. Erben, and E. Kasper, IEEE Electron Device Lett. 13, 206 (1992).

${ }^{10}$ R. Oberhuber, G. Zandler, and P. Vogl, Phys. Rev. B 58, 9941 (1998).

${ }^{11}$ S. Verdonckt-Vandebroek, E. F. Crabbe, B. S. Meyerson, D. L. Harame, P. J. Restle, J. M. C. Stork, and J. B. Johnson, IEEE Trans. Electron Devices 41, 90 (1994).

${ }^{12}$ T. Mizuno, S. Takagi, N. Sugiyama, H. Satake, A. Kurobe, and A. Toriumi, IEEE Electron Device Lett. 21, 230 (2000).
${ }^{13}$ D. Marris, A. Cordat, D. Pascal, A. Koster, E. Cassan, L. Vivien, and S. Laval, IEEE J. Sel. Top. Quantum Electron. 9, 747 (2003).

${ }^{14}$ O. Qasaimeh, J. Singh, and P. Bhattacharya, IEEE J. Quantum Electron. 33, 1532 (1997).

${ }^{15}$ D. J. Paul, Semicond. Sci. Technol. 19, R75 (2004).

${ }^{16}$ J. C. Tsang, P. M. Mooney, F. Dacol, and J. O. Chu, J. Appl. Phys. 75, 8098 (1994).

${ }^{17}$ T. S. Perova, R. A. Moore, K. Lyutovich, M. Oehme, and E. Kasper, Thin Solid Films 517, 265 (2008)

${ }^{18}$ H. K. Shin, D. J. Lockwood, and J.-M. Baribeau, Solid State Commun. 114, 505 (2000).

${ }^{19}$ P. Dobrosz, S. J. Bull, S. H. Olsen, and A. G. O'Neill, Surf. Coat. Technol. 20, 1755 (2005)

${ }^{20}$ F. Pezzoli, E. Bonera, E. Grilli, M. Guzzi, S. Sanguinetti, D. Chrastina, G. Isella, H. von Känel, E. Wintersberger, J. Stangl, and G. Bauer, Mater. Sci. Semicond. Process. 11, 279 (2008).

${ }^{21}$ J. Schmidt, G. Vogg, F. Bensch, S. Kreuzer, P. Ramm, S. Zollner, R. Liu, and P. Wennekers, Mater. Sci. Semicond. Process. 8, 267 (2005).

${ }^{22}$ J. S. Reparaz, A. Bernardi, A. R. Goñi, P. D. Lacharmoise, M. I. Alonso, M. Garriga, J. Novák, and I. Vávra, Appl. Phys. Lett. 91, 081914 (2007).

${ }^{23}$ J. S. Reparaz, A. R. Goñi, A. Bernardi, M. I. Alonso, and M. Garriga, Phys. Status Solidi B 246, 548 (2009).

${ }^{24}$ J. S. Reparaz, A. Bernardi, A. R. Goñi, M. I. Alonso, and M. Garriga, Appl. Phys. Lett. 92, 081909 (2008).

${ }^{25}$ F. Pezzoli, E. Bonera, E. Grilli, M. Guzzi, S. Sanguinetti, D. Chrastina, G. Isella, H. von Känel, E. Wintersberger, J. Stangl, and G. Bauer, J. Appl. Phys. 103, 093521 (2008).

${ }^{26}$ A. Bernardi, J. S. Reparaz, A. R. Goñi, M. I. Alonso, and M. Garriga, Phys. Status Solidi B 244, 76 (2007).

${ }^{27}$ M. A. Renucci, J. B. Renucci, and M. Cardona, in Light Scattering in Solids (Flammarion, Paris, 1971), p. 326.

${ }^{28}$ M. I. Alonso and K. Winer, Phys. Rev. B 39, 10056 (1989).

${ }^{29}$ V. A. Volodin, M. D. Efremov, A. S. Deryabin, and L. V. Sokolov, Semiconductors 40, 1314 (2006).

${ }^{30}$ J. Humlicek, M. Garriga, M. I. Alonso, and M. Cardona, J. Appl. Phys. 65, 2827 (1989).

${ }^{31}$ J. F. Nye, Physical Properties of Crystals (Clarendon, Oxford, 1985).

${ }^{32}$ J. P. Dismukes, L. Ekstrom, and R. J. Paff, J. Phys. Chem. 68, 3021 (1964).

${ }^{33} \mathrm{~F}$. Schäffler, Properties of Advanced SemiconductorMaterials GaN, AlN, InN, BN, SiC, SiGe, edited by M. E. Levinshtein, S. L. Rumyantsev, and M. S. Shur (Wiley, New York, 2001), pp. 149-188.

${ }^{34}$ R. Schorer, E. Friess, K. Eberl, and G. Abstreiter, Phys. Rev. B 44, 1772 (1991).

${ }^{35}$ For a general discussion of phonon modes in semiconductor alloys within the percolation scheme, see O. Pagès, J. Souhabi, A. V. Postnikov, and A. Chafi, Phys. Rev. B 80, 035204 (2009).

${ }^{36}$ Y. M. Chang, C. L. Dai, T. C. Cheng, and C. W. Hsu, Appl. Surf. Sci. 254, 3105 (2008).

${ }^{37}$ J. Tersoff, Phys. Rev. Lett. 77, 2017 (1996).

${ }^{38}$ E. Ko, M. Jain, and J. R. Chelikowsky, J. Chem. Phys. 117, 3476 (2002).

${ }^{39}$ M. Z. Hossain and H. T. Johnson, J. Appl. Phys. 107, 073515 (2010).

${ }^{40} \mathrm{We}$ point out that to the best of our knowledge the pressure derivative of the mode frequencies and, thus, the strain-shift coefficients do not exhibit any dependence on the compositional inhomogeneities. Moreover, there is a very good agreement between the values of the strain-shift coefficients determined recently but independently by us (Ref. 24) and by Pezzoli et al. (Ref. 25). 